PEB Échanges, Programme pour la construction et l'équipement de l'éducation 2008/02

\title{
Nouvelle-Zélande : la modernisation des établissements scolaires dans un cadre décentralisé
} Bruce Sheerin 
Nouvelle-Zélande : la modernisation des établissements scolaires dans un cadre décentralisé

\section{Par Bruce Sheerin, ministère de l'Éducation, Nouvelle-Zélande}

En Nouvelle-Zélande, les autorités nationales délèguent à chaque établissement d'enseignement le pouvoir de décision en matière de gestion de ses propres actifs. Un tel cadre décentralisé représente un réel défi pour les chefs d'établissement et l'administration scolaire, qui peinent à obtenir l'information sur des sujets complexes liés à la conception de leur établissement. Afin d'apporter cette information aux acteurs concernés, le site Internet du ministère de l'Éducation propose de nombreuses publications sur ces thèmes et offre des exemples de bonnes pratiques.

\section{PRISE DE DÉCISION AU NIVEAU LOCAL}

Depuis 19891', les établissements scolaires néo-zélandais détiennent la responsabilité pour l'entretien de leurs locaux et, depuis 2000, pour toutes les décisions liées à leurs actifs. Le conseil d'administration de chaque établissement (principalement constitué de représentants élus des parents d'élèves) est financé par un budget sur cinq ans et a pour fonction de prendre en charge l'entretien, les agrandissements et les rénovations des bâtiments scolaires. Le principe est que les acteurs de l'éducation les plus proches du terrain sont également les plus à même de créer un environnement optimal pour leurs élèves et enseignants.

Lorsqu'un nouvel établissement doit être construit, le ministère de l'Éducation met en place un conseil d'administration qui travaille avec un concepteur, un chef de projet et une entreprise de construction. Tous ces acteurs font en sorte que le nouvel établissement réponde aux besoins en termes de programmes, de qualité de l'enseignement et de communauté locale. Le ministère s'assure simplement que l'établissement dispose d'un nombre suffisant de salles de classe et que le projet entre dans les limites du budget alloué mais n'intervient pas davantage dans les décisions concernant les détails de la conception de l'établissement.

\section{L'INFORMATION FOURNIE AUX ÉTABLISSEMENTS}

\section{Les publications}

Le ministère propose une série de publications pour aider les établissements à prendre des décisions portant sur la conception intérieure, I'acoustique, le chauffage et l'isolation, l'éclairage, la ventilation et la qualité de l'air. La plupart de ces ouvrages reposent sur des recherches entreprises par la Building Research Association of New Zealand Ltd. (Association néo-zélandaise de recherche sur les bâtiments)

1. Voir OCDE (2004), « Le financement des actifs des établissements en Nouvelle-Zélande », PEB Échanges, n 53, octobre, pp. 7-9. 
ainsi que sur des enquêtes réalisées auprès des membres des conseils d'administration, des chefs d'établissement et des élèves sur leurs attentes en termes de qualité du niveau d'enseignement et de l'environnement d'apprentissage ${ }^{2}$ (www.minedu.govt.nz/goto/classroomdesignsurvey).

Designing Quality Learning Spaces (La conception d'espaces d'apprentissage de qualité) est une publication détaillée et richement illustrée qui s'adresse aux membres des conseils d'administration, aux chefs d'établissement et aux enseignants. En leur apportant une meilleure compréhension des facteurs importants de la conception des établissements et en les aidant à faire en sorte que les professionnels auxquels ils font appel leur délivrent un cadre bâti de grande qualité, ce document assiste les chefs d'établissement dans leur prise de décision éclairée, fondée sur les recherches actuelles, afin d'apporter aux enseignants et aux élèves des environnements scolaires confortables, sains et conçus pour optimiser I'enseignement et I'apprentissage. Cette publication est disponible sur le site Internet du ministère et divisée en cinq fichiers PDF distincts pour un téléchargement facilité (www.minedu.govt.nz/goto/ DesigningLearningSpaces).

\section{Exemples de bonnes pratiques}

Le ministère a également publié des exemples de bonnes pratiques pour montrer à tous les chefs d'établissements, ainsi qu'aux concepteurs engagés pour les aider, la façon dont certains conseils d'administration ont conçu leur établissement ou ont transformé leurs bâtiments existants. Plus de 150 exemples d'espaces d'enseignement et d'apprentissage nouveaux ou modernisés dans 70 établissements scolaires néo-zélandais peuvent être consultés sur le site Internet du ministère (www.minedu.govt.nz/goto/schoolremodels). Parmi ceux-ci, on trouve :

- une vingtaine de nouveaux établissements construits au cours de la dernière décennie, recouvrant les niveaux primaire, du premier et du deuxième cycle du secondaire ;

- des salles de classes modernisées (tous niveaux d'enseignement confondus), le but de l'amélioration étant de créer un espace pour un enseignement du XXIe siècle de qualité et s'adressant à un public varié ;

- des salles spécialisées pour les sciences, les technologies de l'information et des communications et les technologies pédagogiques ;

- des centres de documentation/des bibliothèques ;

- des salles polyvalentes et des espaces destinés aux arts du spectacle ;

- des zones administratives/de réception ;

- des espaces d'apprentissages pour l'éducation sanitaire, physique et sportive ;

- des espaces conçus pour l'interaction sociale des élèves, tels que les « rues d'apprentissage ».

Les exemples sont assortis de photographies, de plans et d'un récit détaillé des différentes démarches que l'établissement a entreprises pour parvenir à ses buts pédagogiques et résultats d'apprentissage, ainsi que de la façon dont l'établissement a aidé à remplir les objectifs du gouvernement en favorisant l'engagement des familles et de la communauté et en proposant un enseignement de qualité. Y sont également décrits le processus de consultation du personnel et des élèves pour l'élaboration des plans de construction, les possibilités d'utilisation des espaces nouveaux ou modernisés par rapport aux possibilités antérieures plus limitées, ainsi que l'attention portée à l'acoustique, la ventilation, le chauffage et l'éclairage.

2. Voir OCDE (2005), « Infrastructures pour l'apprentissage en Nouvelle-Zélande », PEB Échanges, n 56, octobre, pp. 7-8. 


\section{École primaire Papatoetoe South}

L'un de ces exemples est une nouvelle salle ajoutée à l'école primaire Papatoetoe South, à Auckland. Il s'agit d'une école destinée aux niveaux 1 à 6 (c'est-à-dire pour des enfants âgés de 5 à 11 ans) dans une zone à faible revenu et avec un effectif total de 590 élèves. Il fallait à l'établissement un espace pour accueillir 40 élèves et 2 professeurs qui mette en avant l'apprentissage orienté par les élèves euxmêmes et assisté par les technologies.

La nouvelle salle (voir la photo ci-après) comporte des mini-gradins spécialement conçus pour I'environnement scolaire, deux tableaux blancs interactifs, plusieurs ordinateurs, deux salles d'atelier ainsi qu'une grande véranda, des meubles "conformes aux meilleures pratiques ", un système de renouvellement de l'air, un système de champ sonore ainsi qu'un revêtement intérieur favorisant une bonne acoustique.

Le plan simple de la salle (voir ci-après) offre un espace suffisant pour accueillir 40 élèves de 11 ans ainsi que leurs deux enseignants. Les salles d'atelier, auparavant utilisées pour le stockage, hébergent à présent un espace robotique pour aider les élèves dans les exercices de résolution de problèmes ainsi qu'un espace calme pour les petits groupes. De grandes portes s'ouvrent sur une vaste véranda utilisée pour l'apprentissage en intérieur/extérieur.

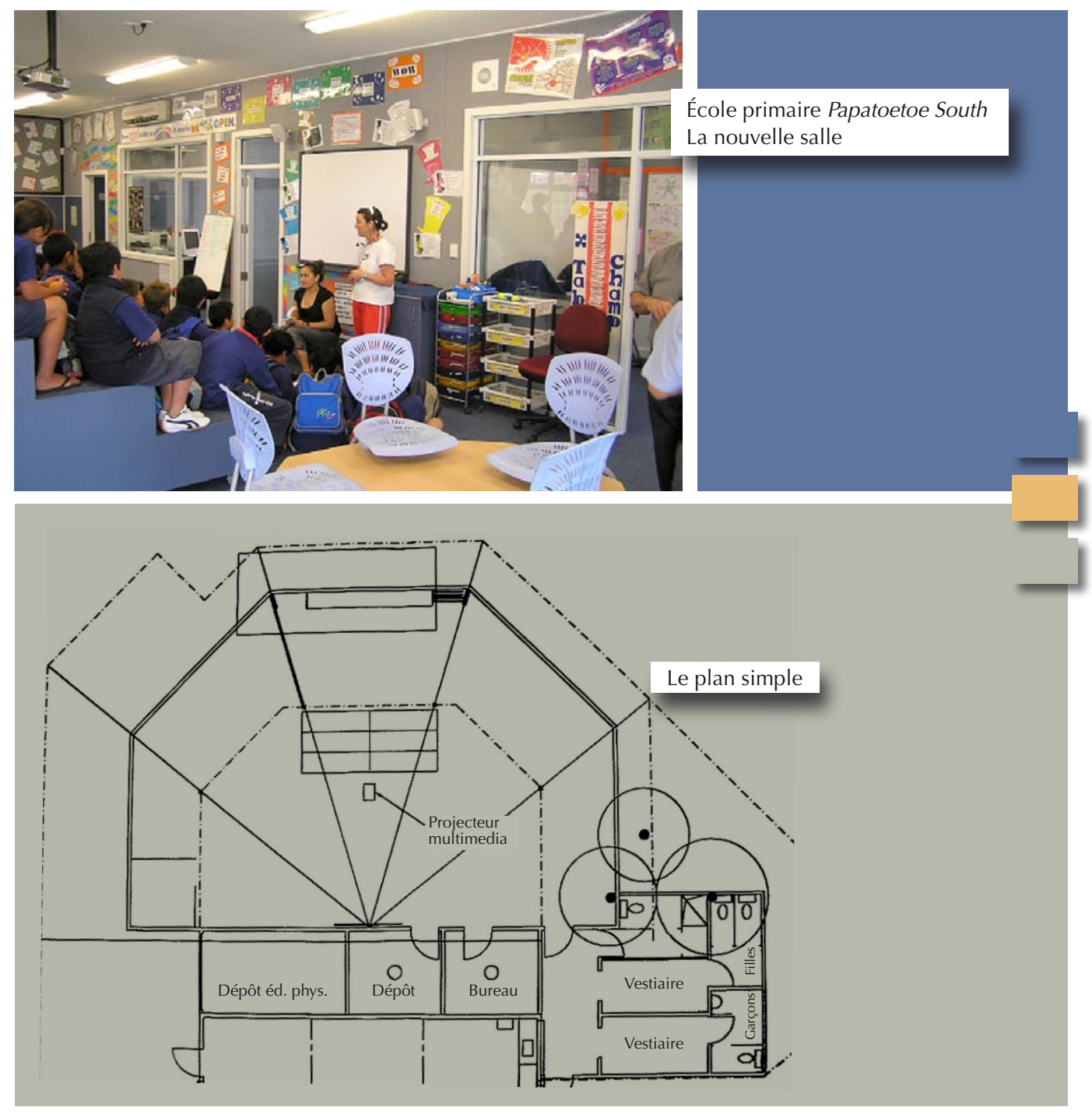


"Nous privilégions les approches d'apprentissage qui donnent aux élèves les outils pour apprendre comment apprendre, et ce tout au long de leur vie », déclare le chef d'établissement, David Dawson. Une telle vision conduit à l'adoption d'un modèle personnalisé, reposant sur des repères graphiques et sur la taxonomie de Bloom ${ }^{3}$, qui donne aux élèves un accès aux technologies et met en avant les compétences en littéracie et les notions de calcul. L'établissement attend de tous ses enseignants qu'ils possèdent un niveau élevé de connaissances en technologies. La formation professionnelle dans ce domaine est d'ailleurs à la pointe : les enseignants vont au Danemark, aux États-Unis et au RoyaumeUni pour développer leurs capacités et plus de la moitié des espaces d'enseignement sont équipés de tableaux blancs interactifs. "Nous attendons de nos enseignants et de nos élèves qu'ils se fixent des objectifs d'apprentissage réalistes; que nos enseignants sachent où se situe chaque élève et comment il évolue dans son apprentissage, et qu'ils soient à même d'aider les élèves à devenir des apprenants autonomes et produtifs. Nous fournissons les espaces et l'équipement qui contribuent à réaliser ces objectifs », affirme David Dawson.

À partir d'exemples comme celui-ci, collectés dans toute la Nouvelle-Zélande, les établissements autonomes peuvent examiner toutes les possibilités qui s'offrent pour des bâtiments nouveaux ou anciens, relever des idées et orienter leurs concepteurs et constructeurs pour obtenir les environnements $d^{\prime}$ enseignement et d'apprentissage qu'ils souhaient pour leurs élèves et leurs enseignants.

Si d'autres pays disposent de sites Internet ou de publications offrant des informations sur la conception et la modernisation de bâtiments scolaires qui puissent être pertinentes pour les établissements néozélandais, le ministère de l'Éducation de la Nouvelle-Zélande serait heureux de proposer les liens correspondants sur son propre site.

Pour en savoir plus, contacter :

Bruce Sheerin

Senior Policy Analyst (Property)

Ministry of Education

45-47 Pipitea Street

Thorndon

Wellington 6140

Nouvelle-Zélande

bruce.sheerin@minedu.govt.nz

www.minedu.govt.nz/performingclassrooms

3. "La taxonomie de Bloom répartit les objectifs éducatifs en trois "domaines" : affectif, psychomoteur et cognitif. [...] L'un des buts de la taxonomie de Bloom est de motiver les acteurs de l'éducation à se focaliser sur ces trois domaines, créant ainsi une forme d'éducation plus "globale" » (traduction de l'article de Wikipedia (version consultée le 17 décembre 2007) disponible en anglais sur: http://en.wikipedia. org/wiki/Taxonomy_of_Educational_Objectives). 


\section{ORGANISATION DE COOPÉRATION ET DE DÉVELOPPEMENT ÉCONOMIQUES}

L'OCDE est un forum unique en son genre où les gouvernements de 30 démocraties œuvrent ensemble pour relever les défis économiques, sociaux et environnementaux que pose la mondialisation. L'OCDE est aussi à l'avant-garde des efforts entrepris pour comprendre les évolutions du monde actuel et les préoccupations qu'elles font naître. Elle aide les gouvernements à faire face à des situations nouvelles en examinant des thèmes tels que le gouvernement d'entreprise, l'économie de l'information et les défis posés par le vieillissement de la population. L'Organisation offre aux gouvernements un cadre leur permettant de comparer leurs expériences en matière de politiques, de chercher des réponses à des problèmes communs, d'identifier les bonnes pratiques et de travailler à la coordination des politiques nationales et internationales.

Les pays membres de l'OCDE sont: l'Allemagne, l'Australie, l'Autriche, la Belgique, le Canada, la Corée, le Danemark, l'Espagne, les États-Unis, la Finlande, la France, la Grèce, la Hongrie, l'Irlande, l'Islande, l'Italie, le Japon, le Luxembourg, le Mexique, la Norvège, la Nouvelle-Zélande, les Pays-Bas, la Pologne, le Portugal, la République slovaque, la République tchèque, le Royaume-Uni, la Suède, la Suisse et la Turquie. La Commission des Communautés européennes participe aux travaux de l'OCDE.

Les Éditions OCDE assurent une large diffusion aux travaux de l'Organisation. Ces derniers comprennent les résultats de l'activité de collecte de statistiques, les travaux de recherche menés sur des questions économiques, sociales et environnementales, ainsi que les conventions, les principes directeurs et les modèles développés par les pays membres.

Cet ouvrage est publié sous la responsabilité du Secrétaire général de l'OCDE. Les opinions et les interprétations exprimées ne reflètent pas nécessairement les vues de l'OCDE ou des gouvernements de ses pays membres.

Les corrigenda des publications de l'OCDE sont disponibles sur : www.oecd.org/editions/corrigenda. (C) OCDE 2008

Toute reproduction, copie, transmission ou traduction de cette publication doit faire l'objet d'une autorisation écrite. Les demandes doivent être adressées aux Éditions OCDE rights@oecd.org ou par fax 331452499 30. Les demandes d'autorisation de photocopie partielle doivent être adressées au Centre français d'exploitation du droit de copie (CFC), 20, rue des Grands-Augustins, 75006 Paris, France, fax 33146346719 , contact@cfcopies.com ou (pour les États-Unis exclusivement) au Copyright Clearance Center (CCC), 222 Rosewood Drive, Danvers, MA 01923 , USA, fax 19786468600 , info@copyright.com. 